\title{
In vitro cytotoxicity of hydrogels based on chitosan and modified with gold nanoparticles
}

\author{
Bożena Tyliszczak ${ }^{1}$ - Anna Drabczyk ${ }^{2}$ - Sonia Kudlacik-Kramarczyk ${ }^{2}$. \\ Katarzyna Bialik-Wąs ${ }^{3} \cdot$ Agnieszka Sobczak-Kupiec $^{2}$
}

Received: 30 April 2017 / Accepted: 20 August 2017 / Published online: 1 September 2017

(C) The Author(s) 2017. This article is an open access publication

\begin{abstract}
Hydrogels belong to the group of polymers that recently become more and more popular. This is a result of unique properties of the mentioned materials such as biocompatibility, biodegradability as well as a great sorption capacity. Combination of such polymers with gold nanoparticles results in the preparation of innovative materials that can be applicable in wide range of areas with special emphasis on medicine. In present publication series of chitosan based hydrogels modified with gold nanoparticles were prepared. These materials were subsequently characterized by a number of physicochemical analysis concerning determination of cytotoxicity to epidermal cells. Based on these research it can be concluded that hydrogels containing small quantities of gold nanoparticles do not exhibit toxicity in relation to these cells. Furthermore, it can be said that addition of nanogold into hydrogel resulted in a decrease of its porosity as well as in a slight decrease of the sorption capacity of materials tested. Prepared polymers were characterized by biocompatibility in relation to simulated body fluid. Proposed hydrogels constitute an interesting type of materials that could be potentially considered for biomedical use.
\end{abstract}

Bożena Tyliszczak

btyliszczak@chemia.pk.edu.pl

Anna Drabczyk

adrabczyk@chemia.pk.edu.pl

1 Department of Chemistry and Technology of Polymers, Cracow University of Technology, Warszawska 24, 31-155 Cracow, Poland

2 Institute of Inorganic Chemistry and Technology, Cracow University of Technology, Warszawska 24, 31-155 Cracow, Poland

3 Institute of Organic Chemistry and Technology, Cracow University of Technology, Warszawska 24, 31-155 Cracow, Poland
Keywords Chitosan · Hydrogels · Nanogold · Cytotoxicity · Epidermal cells

\section{Introduction}

Hydrogels belong to the group of biocompatible, biodegradable, non-toxic polymers widely applied in medicine and pharmacy. Physicochemical properties of these materials depend on the type of compound used for a hydrogel matrix preparation. For synthesis of hydrogels polymers such as poly(acrylic acid), poly(vinyl alcohol), poly(2-hydroxyethyl methacrylate) or poly(ethylene glycol) are applied [1, 2]. The most popular is poly(acrylic acid). However, this organic compound is corrosive and has a harmful impact on the environment [3]. Therefore, some alternative materials that could constitute a hydrogel matrix have been considered.

Attention has been directed to the application of compounds belonging to the group of proteins or polysaccharides. One of them is chitosan. It is a naturally occurring polysaccharide that is characterized by biodegradability and low toxicity [4]. Moreover, versatility and biological compatibility are very interesting features of the described biopolymer. Besides, from a medical point of view, chitosan does not provoke an immune response. It is worth underlining that discussed material is the second, after cellulose, most plentiful biopolymer of natural origin. Due to all mentioned above properties of polysaccharide, chitosan could be applied as a component of a system used for drug delivery administered by oral or subcutaneous injection [5, 6]. Recently, Bhattarai [7] reported an application of hydrogels derived from chitosan as systems for controlled, localized drug delivery. Described polysaccharide, a material that does not cause inflammation and is not characterized by an immunogenicity, can be used for 
subcutaneous drug delivery systems [8]. Hydrogels based on chitosan have been also used in the antitumor therapy. Hydrogels are loaded with radioisotopes and then inserted into the place affected by cancer that is subsequently treated with an X-ray [9]. The use of such a system slows down the growth and progression of tumor, and in most cases prevents its metastasis. That was demonstrated by conducting studies on mice with breast cancer. Such a system has been also used in therapy against a tumor of the brain [10]. Moreover, hydrogels based on chitosan have also been used in the form of dressings stimulating acute wound healing. Such polymers have been applied for corneal wound healing [11-13]. Currently, many studies aim at preparation of composites of chitosan and nanomaterials. Such materials could be potentially used in many areas [14-17].

The aim of the present work was a modification of hydrogels based on chitosan by inserting nanogold particles. Nanogold particles are known from their antibacterial properties $[18,19]$. This form of gold is widely used in the pharmacy and cosmetology. Furthermore, due to their ability to absorb radiation from the infrared range, these nanoparticles are applied in photo-thermal therapy used in cancer treatment. Gold nanoparticles have been also used as contrast agents and drug carriers [20-22].

\section{Materials}

Chitosan, poly(ethylene glycol) diacrylate, hydrogen tertachloroaurate (III) trihydrate, sodium borohydride and 2hydroxy-2-methylpropiophenon used for hydrogels preparation were supplied by Sigma Aldrich while gelatin and trisodium citrate were received from Avantor Performance Materials (formerly POCH SA). All compounds were characterized by analytical purity and applied without further treatment.

\section{Experimental part}

\section{Preparation of gold nanoparticles}

Gold nanoparticles were obtained using chemical process [23] of reduction of hydrogen tertachloroaurate (III) trihydrate with sodium borohydride in the presence of trisodium citrate, acting as reducing and stabilizing agents [24]. Resulted concentration of gold nanoparticles amounted to $250 \mathrm{ppm}$.

\section{Synthesis of hydrogels containing gold nanoparticles}

Hydrogels on the basis of chitosan and modified with nanoparticles of gold have been synthesized using UV radiation.
The main synthesis consisted of several stages. Indicated in Table 1 amounts of chitosan and gelatin were dissolved in $0.05 \%$ solution of acetic acid and then a required quantity of solution containing gold nanoparticles (at a concentration of $250 \mathrm{ppm})$ was added to the whole solution. Then, $8 \mathrm{ml}$ of poly(ethylene glycol) diacrylate (molecular weight $\mathrm{Mn}$ equal $700 \mathrm{~g} / \mathrm{mol}$ ) as a crosslinking agent and finally $0.25 \mathrm{ml}$ of 2hydroxy-2-methylpropiophenone as a photoinitiator were added. The whole mixture was subsequently exposed to UV radiation. Final materials were dried at room temperature and subjected to further analyses. Compositions of chitosan based hydrogels modified with gold nanoparticles are presented in Table 1.

In Fig. 1 photography of prepared hydrogel sample is presented.

Such samples were subsequently subjected to further research in order to determine their physico-chemical properties.

\section{UV-Vis spectroscopy}

UV-Vis spectroscopy measurements $(330-700 \mathrm{~nm})$ were performed using a Specord 205 at room temperature with a $1-\mathrm{cm}$ optical length cuvette and a spectral resolution of $1 \mathrm{~nm}$.

\section{Studies on sorption capacity}

For this purpose dried and weighed materials were immersed respectively in distilled water and in simulated body fluid ( $\mathrm{SBF}$, solution at $\mathrm{pH}=7.40$ ) composed of ions having concentrations close to concentrations of ions present in human blood plasma). Samples, after immersion in solutions for 1, 24 or $72 \mathrm{~h}$, were separated from the solution in the swollen state and weighed. Sorption capacity was determined by swelling ratio $(\mathrm{Q}, \mathrm{g} / \mathrm{g})$ calculated from the equation:

$Q=\frac{w-w_{0}}{w_{0}}$,

where:

$\mathrm{w}$ - hydrogel weight in a swollen state $[\mathrm{g}], \mathrm{w}_{0}$ - hydrogel weight before swelling [g].

\section{Incubation studies}

Incubation studies have been carried out in order to determine a behavior of synthesized materials in selected liquids by checking $\mathrm{pH}$. Samples of hydrogels were incubated in distilled water and in simulated body fluid (SBF) for a period of about three weeks. Research was conducted at $37^{\circ} \mathrm{C}$. $\mathrm{pH}$ value was measured every two days. 
Table 1 Compositions of prepared hydrogels

\begin{tabular}{llllll}
\hline No. & $\begin{array}{l}\text { Gelatin } 2 \% \\
{[\mathrm{ml}]}\end{array}$ & $\begin{array}{l}\text { Chitosan 3\% } \\
{[\mathrm{ml}]}\end{array}$ & $\begin{array}{l}\text { Gold nanoparticles } \\
{[\mathrm{ml}]}\end{array}$ & $\begin{array}{l}\text { Crosslinking agent } \\
{[\mathrm{ml}]}\end{array}$ & $\begin{array}{l}\text { Photoinitiator } \\
{[\mathrm{ml}]}\end{array}$ \\
\hline 1. & 20 & 30 & 0 & 8 & 0.25 \\
2. & 20 & 30 & 1 & 8 & 0.25 \\
3. & 20 & 30 & 3 & 8 & 0.25 \\
4. & 20 & 30 & 5 & 8 & 0.25 \\
\hline
\end{tabular}

\section{Cytotoxicity studies}

Evaluation of cytotoxicity of hydrogel materials was conducted by means of XTT and MTT assays according to the Polish standards PN-EN ISO 10993-5:2009 using epidermal CD 1106 KERTr cell lines. Prior to this analysis, extracts from hydrogels (100, 75, 50 and $25 \mathrm{wt} \%$ ) were prepared according to the Polish standard PN-EN ISO 10993$12: 2012$.

The MTT cytotoxicity test based on reduction of 3-(4,5dimethyl-2-thiazolyl)-2,5-diphenyl-2H-tetrazoline bromide (known as MTT reagent) to form azure formazan in living cells enables to evaluate enzymatic activity of the succinate dehydrogenase thereby to determine the cellular vitality. Subsequently, amount of formazan formed adequately to the amount of living cells is determined by colorimetric measurements.

Determination of cytotoxicity using XTT assay is based on evaluation of enzymatic activity of mitochondrial dehydrogenase-enzyme that is active only in the living cells. The (2,3-bis(2-methoxy-4nitro-5-sulphophenyl)5-[phenylamine)carbonyl]-2H tetrazolim hydroxide), defined as XTT reagent, after being added to the cell culture, undergoes reduction to azure formazan by mitochondrial dehydrogenase present in the living cells. Subsequently, the amount of formazan that determines the amount of living cells is defined by colorimetric measurements.

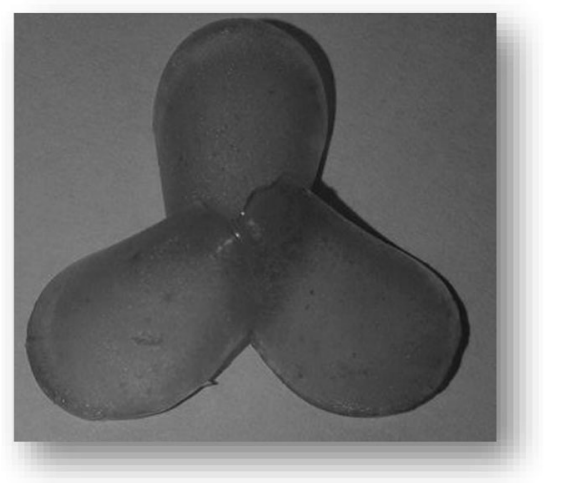

Fig. 1 Photography of hydrogel samples modified with gold nanoparticles $(3 \mathrm{ml})$

\section{Scanning electron microscopy (SEM)}

SEM (Scanning Electron Microscopy) analyses were performed using Helios NanoLab H50HP equipment. Sputtering samples with carbon was performed at an uniform rate. The carbon layer was approximately $15 \mathrm{~nm}$.

\section{Results and discussion}

\section{UV-Vis analysis}

The presence of nanogold suspension was confirmed by UVVis spectroscopy (Fig. 2). The optical response of metal nanoparticles is often characterized by the presence of a strong absorption peak that is usually absent in the corresponding spectrum of the bulk metal. This is attributed to a resonance in the collective motion of the conduction electrons in response to an electromagnetic field and is called the localized surface plasmon resonance.

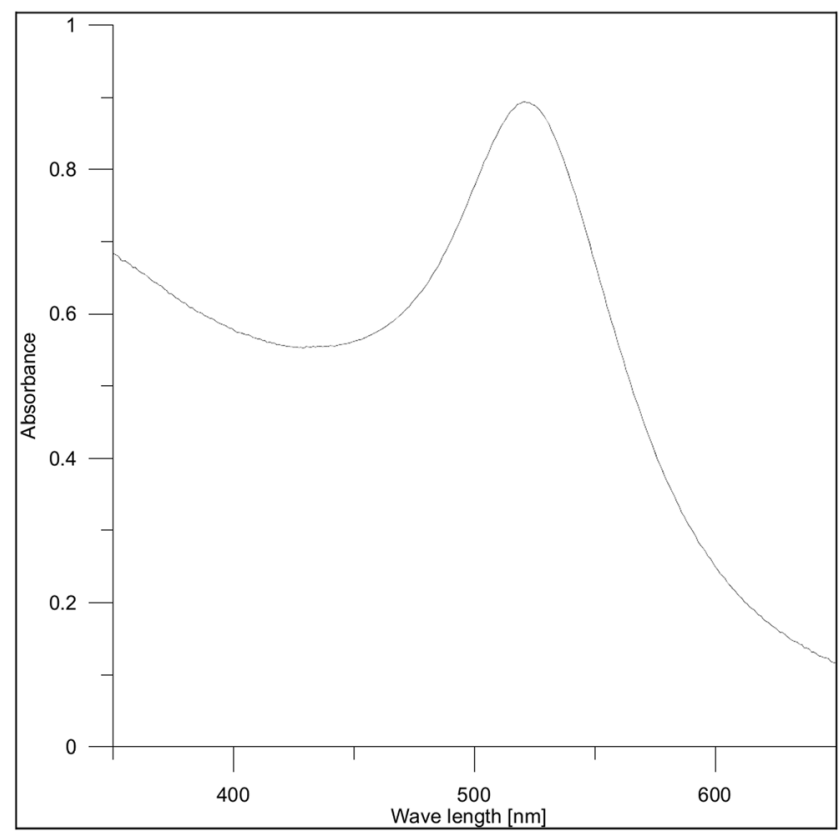

Fig. 2 UV-Vis spectrum of obtained nanogold 
According to the literature the distinct absorption peak from the surface plasmon of the gold nanoparticles should be located between 510 and $530 \mathrm{~nm}$ [20]. In the obtained UV-Vis spectrum (Fig. 2) the maximum absorbance has been recorded at $521 \mathrm{~nm}$. This result indicates that by chemical process [21], based on the reduction of hydrogen tertachloroaurate (III) trihydrate with sodium borohydrate in the presence of trisodium citrate, nanogold particles have been synthesized.

\section{Sorption capacity of hydrogels}

Sorption capacity calculated from swelling ratio after immersion of samples in selected liquids has been presented in Fig. 3.

Swelling process involves absorption of liquid by a material immersed. Hydrogels are materials that are characterized by a particularly large sorption capacity. That is related to the chemical structure with large amount of crosslinked polymer chains. In a dry state this structure is tightly coiled, while in contact with solvent undergoes changes. Water penetrates into the interior of the hydrogel network, and interacting with hydrophilic groups results in their dissociation. Therefore, formation of ions with numerous electrostatic interactions such as repulsion increases the distance between the polymer

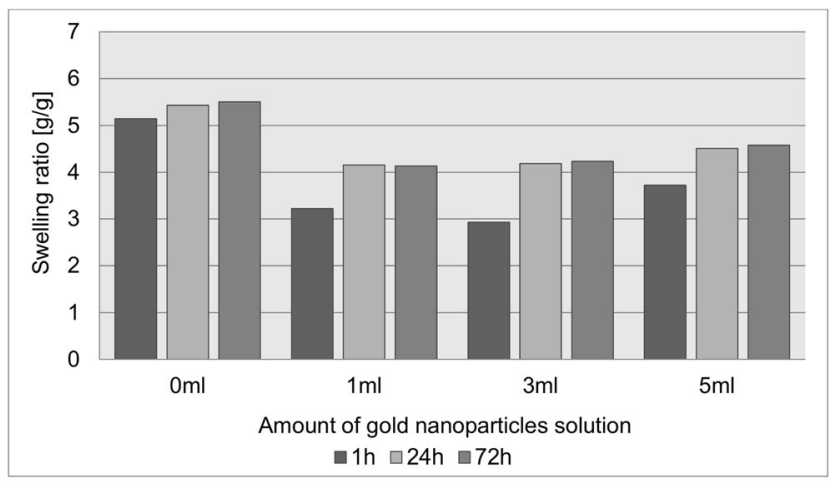

a)

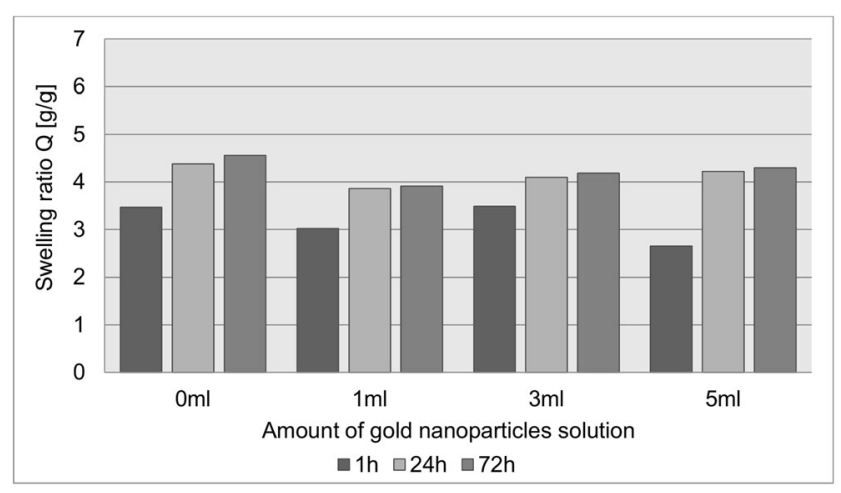

b)

Fig. 3 Sorption capacity of hydrogels modified with gold nanoparticles in $\mathbf{a}$ distilled water and $\mathbf{b}$ simulated body fluid (SBF) chains and consequently results in loosening of the polymer network.

All prepared hydrogels were characterized by relatively high swelling ability. Introduction of nanogold into hydrogel matrix resulted in slight decrease of sorption capacity - swelling ratios calculated for unmodified hydrogels had slightly greater values in the case of the majority of samples. It is probably a consequence of chelation of some functional groups by gold nanoparticles in the polymer network. Chelation of the hydrophilic groups restrains their interactions with water, thus hampers a loosening of polymer network. Therefore, higher sorption capacity was observed for hydrogels before addition of gold nanoparticles.

However, any relationship between swelling sorption and the amount of introduced nanogold was not observed due to the fact that hydrogels modified with gold nanoparticles suspension were characterized by a comparable swelling ability in both tested solutions.

It is worth mentioning that the lowest sorption capacity was observed after $1 \mathrm{~h}$ immersion. The swelling ability of the material was higher after $24 \mathrm{~h}$ and maintained on the same level for $72 \mathrm{~h}$.

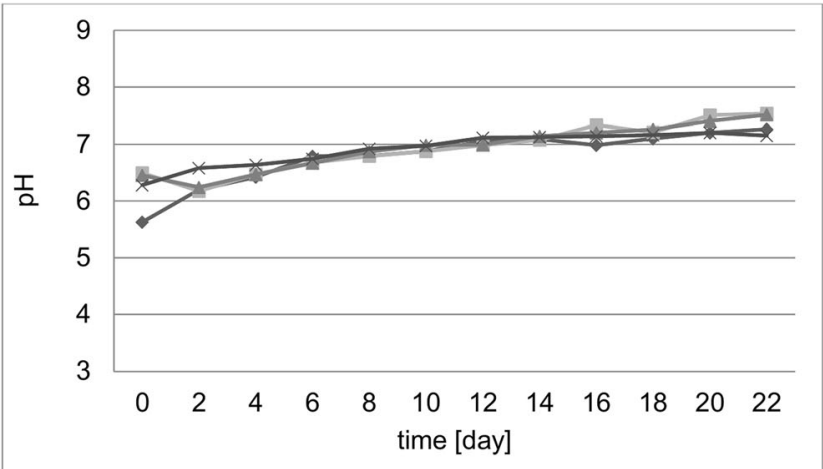

$\multimap 0 \mathrm{ml}$ nanoAu $-1 \mathrm{ml}$ nanoAu $\longleftarrow 3 \mathrm{ml}$ nanoAu $\varkappa 5 \mathrm{ml}$ nanoAu

a)

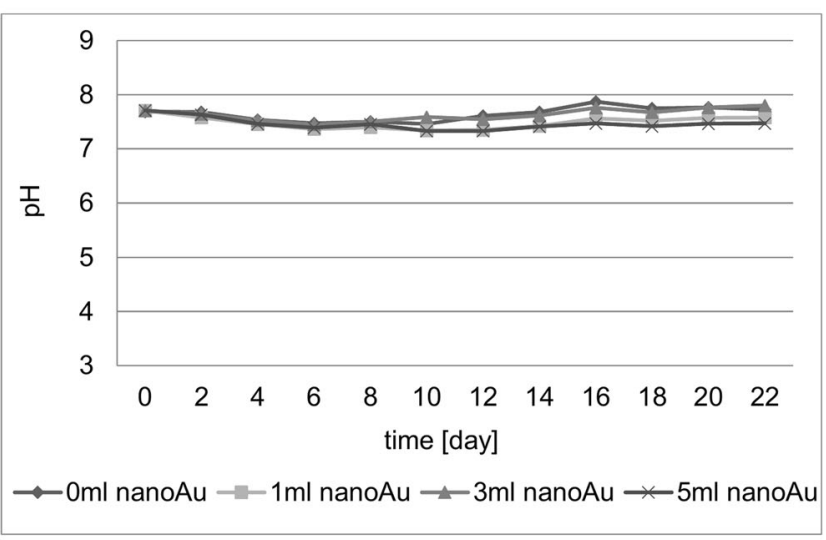

b)

Fig. 4 Results of $\mathrm{pH}$ measurements in a distilled water and $\mathbf{b} \mathrm{SBF}$ 
Table 2 MTT assay of the hydrogel extracts exposed to the epidermal cell lines

\begin{tabular}{clllll}
\hline \multirow{2}{*}{ Nanogold [ml] } & & \multicolumn{2}{l}{ Extract concentration [wt.\%] } & \multicolumn{2}{c}{ Cytotoxicity } \\
\cline { 3 - 5 } & 100 & 75 & 50 & 25 & \\
\hline 1 & 76.7 & 93.8 & 101.8 & 117.5 & No \\
3 & 128.1 & 131.6 & 134.0 & 129.1 & No \\
5 & 29.6 & 33.9 & 39.5 & 54.8 & Yes \\
\hline
\end{tabular}

\section{Incubation studies}

Results of incubation of the hydrogels in solutions have been presented in Fig. 4. pH measurement in distilled water indicated that some balance between the interior of the hydrogel and the fluid in which the samples were immersed was possibly happening.

Dry hydrogel after introducing into the distilled water begins to swell. Water absorption by the sample results in an elution of unreacted reagents. As mentioned above, the presence of gold nanoparticles restrains the interactions between the solution and the functional groups of the polymer network. Thus, limited absorption and limited elution were observed in case of samples modified with gold nanoparticles. During the consecutive days of incubation $\mathrm{pH}$ was maintaining at a comparable level oscillating around value of 7.0. At the end of the incubation time (22 days) $\mathrm{pH}$ value was 7.5.

In case of samples immersed in SBF $\mathrm{pH}$ values were also continuing to maintain at a very similar level and, what is important, fluctuations in $\mathrm{pH}$ values were smaller in comparison to those measured in distilled water. $\mathrm{pH}$ values measured in SBF solution quickly stabilized and established at the same level. It is very interesting and important information on the basis of which it is possible to attempt to try to determine the tested materials as biocompatible. Such information allows to further investigation of the obtained hydrogels for medical use. Moreover, conducted studies also give an information that during the incubation process a degradation of immersed samples was not observed. In the course of studies spikes or decreases of the $\mathrm{pH}$ values, which could indicate a degradation of the hydrogel, were not recorded.

\section{Evaluation of cytotoxicity}

- Determination of the cytotoxicity of hydrogel extracts towards epidermal cells using MTT assay.

Results of viability of epidermal cells (CCD 1106 KERTr) after $24 \mathrm{~h}$ culture in the presence of the extracts from the tested hydrogels prepared using the substrate based on KeratinocyteSerum Free Medium (Gibco 17,005-042) are presented in Table 2. The research was conducted according to the MTT assay.

- Determination of the cytotoxicity of hydrogel extracts towards epidermal cells using XTT assay.

The results of viability of epidermal cells (CCD 1106 KERTr) after $24 \mathrm{~h}$ culture in the presence of the extracts from the tested hydrogels made of the substrate based on Keratinocyte - Serum Free Medium (Gibco 17,005-042) are shown in Table 3. The research was conducted with the use of MTT assay.

Studies on cytotoxicity revealed that in case of the majority of samples tested any negative impact on epidermal cell was not observed. Based on the results of both XTT and MTT tests regardless on the extract concentration it can be concluded that hydrogels modified with 1 and $3 \mathrm{ml}$ are not characterized by cytotoxicity to tested type of cells. However, hydrogels containing the highest amount of additive $(5 \mathrm{ml})$ exhibit cytotoxicity. Probably it is due to the fact that nanogold solution in such amount has a negative impact on the cells however its significantly smaller amount does not affect the cells' survival. Therefore, sample with 1 and $3 \mathrm{ml}$ will be subjected in the future to the more advanced studies.
Table 3 XTT assay of the hydrogel extracts exposed to the epidermal cell lines

\begin{tabular}{llllll}
\hline \multirow{2}{*}{ Nanogold [ml] } & & \multicolumn{2}{l}{ Extract concentration [wt.\%] } & & \multirow{2}{*}{ Cytotoxicity } \\
\cline { 3 - 4 } & 100 & 75 & 50 & 25 & \\
\hline 1 & 71.0 & 64.3 & 84.4 & 77.4 & No \\
3 & 86.9 & 87.5 & 100.4 & 104.9 & No \\
5 & 38.6 & 60.5 & 67.9 & 89.9 & Yes \\
\hline
\end{tabular}


Fig. 5 SEM images of hydrogels obtained without nanogold (a) and modified with this additive (b)

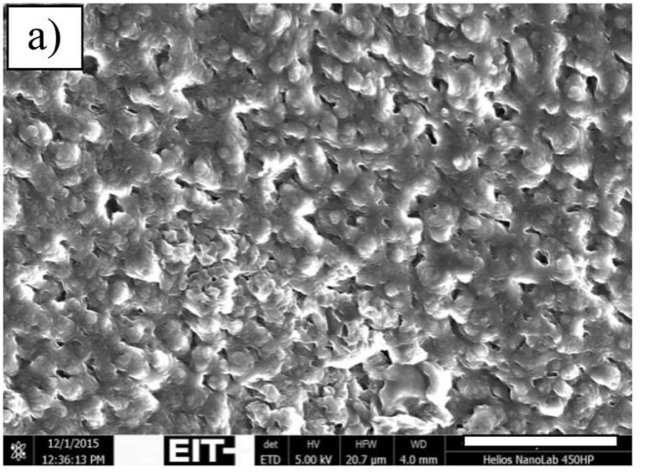

\section{SEM images}

SEM images presented in Fig. 5 illustrate surface morphology of the polymer matrix without any additive (Fig. 5a) and the polymer containing $3 \mathrm{ml}$ of nanogold (Fig. $5 \mathrm{~b}$ ).

On the basis of SEM microphotographs it can be concluded that introduction of nanogold into hydrogel matrix clearly affects the surface morphology of the material obtained. The structure becomes less porous, more smooth and homogeneous. Modification of the hydrogel matrix with an excessive amount of additive would result in a significant reduction in the porosity of the synthesized material. That would be not beneficial due to the possibility of total disorder of physicochemical properties of the material including the swelling capacity.

\section{Conclusions}

A series of hydrogels based on chitosan containing gold nanoparticles have been obtained using UV radiation. Synthesized materials are characterized by a relatively low sorption capacity, which may be caused by chelation of functional groups by gold nanoparticles in the polymer matrix. Hydrogels obtained do not undergo the process of degradation in simulated body fluid. During incubation studies any sudden changes in the $\mathrm{pH}$ values of the solutions, which would be caused by a decomposition of immersed samples, were not observed.

On the basis of evaluation of cytotoxicity it was concluded that the most favorable results were obtained for hydrogels containing $3 \mathrm{ml}$ of gold nanoparticles solution in $50 \mathrm{ml}$ of polymer mixture. After 24-h exposure to the cells of epidermis, the majority of the tested materials have not demonstrated any cytotoxic properties. Hydrogel materials containing the highest amount of gold nanoparticles solution negatively affected on the tested cells in relation to the control cells - the value assigned by PN-EN ISO 10993-5: 2009. Therefore it can be concluded that hydrogels with smaller quantities of nanogold can be subjected to the more advanced studies. Furthermore, SEM analysis indicated that the addition of gold nanoparticles into the hydrogel resulted to some extent in reduction of the porosity of the material. In comparison to the sample without additives, those containing nanogold particles were characterized by higher homogeneity and smoothness. Present hydrogels constitute a very interesting materials for further studies and affect the development of fields such as nanotechnology or polymer technology as well as are potential components for the preparation of modern wound dressings.

Acknowledgements The authors would like to thank The National Centre for Research and Development (Grant no: LIDER/033/697/L-5/ $13 / \mathrm{NCBR} / 2014$ ) for providing financial support to this project.

Open Access This article is distributed under the terms of the Creative Commons Attribution 4.0 International License (http:// creativecommons.org/licenses/by/4.0/), which permits unrestricted use, distribution, and reproduction in any medium, provided you give appropriate credit to the original author(s) and the source, provide a link to the Creative Commons license, and indicate if changes were made.

\section{References}

1. Hoffman AS (2002) Hydrogels for biomedical applications. Adv Drug Deliv Rev 54:3-12

2. Tyliszczak B, Pielichowski K (2007) Charakterystyka matryc hydrożelowych - zastosowania biomedyczne superabsorbentów polimerowych. Czas Tech 104(1):159-167

3. Sverdrup L, Kallqvist T, Kelley AE, Furst CS, Hagen B (2001) Comparative toxicity of acrylic acid to marine and freshwater microalgae and the significance for environmental effects assessments. Chemosphere 45(4-5):653-658

4. Mucha M (2010) Chitozan - wszechstronny polimer ze źródeł odnawialnych. WNT, Warszawa,

5. Kumar MN, Muzzarelli RA, Muzzarelli C, Sashiwa H, Domb AJ (2004) Chitosan chemistry and pharmaceutical perspectives. Chem Rev 104:6017-6084

6. Kean T, Thanou M (2010) Biodegradation, biodistribution and toxicity of chitosan. Adv Drug Deliv Rev 62(1):3-11

7. Bhattarai N, Gunn J, Zhang M (2010) Chitosan-based hydrogels for controlled, localized drug delivery. Adv Drug Deliv Rev 62(1):8399

8. Khor E, Lim LY (2003) Implantable applications of chitin and chitosan. Biomaterials 24:2339-2349

9. Azab AK, Kleinstern J, Doviner V, Orkin B, Srebnik M, Nissan A, Rubinstein A (2007) Prevention of tumor recurrence and distant metastasis formation in a breast cancer mouse model by 
biodegradable implant of 131I-norcholesterol. J Control Release 123:116-122

10. Lesniak MS, Brem H (2004) Targeted therapy for brain tumors. Nat Rev Drug Discov 3:499-508

11. Azad AK, Sermsintham N, Chandrkrachang S, Stevens WF (2004) Chitosan membrane as a wound-healing dressing: characterization and clinical application. J Biomed Mater Res B Appl Biomater 69: 216-222

12. Tsai CY, Woung LC, Yen JC, Tseng PC, Chiou SH, Sungy J, Liu KT, Cheng YH (2016) Thermosensitive chitosan-based hydrogels fur sustained release of ferulic acid on corneal wound healing. Carbohydr Polym 135(1):308-315

13. Tyliszczak B, Walczyk D, Wilczyński S (2015) Acrylic hydrogels modified with bee pollen for biomedical applications. J Appl Pharm Sci 5:010-014

14. Liu M, Wu C, Jiao Y, Xiong S, Zhou C (2013) Chitosanhalloysite nanotubes nanocomposite scaffolds for tissue engineering. J Mater Chem B 1(15):2078-2089

15. Liu M, Zhang Y, Wu C, Xiong S, Zhou C (2012) Chitosan/ halloysite nanotubes bionanocomposites: structure, mechanical properties and biocompatibility. Int J Biol Macromol 51(4):566575

16. Liu M, Ao P, Zhou C, Shen Y, Dai L, Liu Z (2014) The improvement of hemostatic and wound healing property of chitosan by halloysite nanotubes. RSC Adv 4(45):23540-23553

17. Liu M, Chang Y, Yang J, You Y, He R, Chen T, Zhou C (2016) Functionalized halloysite nanotube by chitosan grafting for drug delivery of curcumin to achieve enhanced anticancer efficacy. J Mater Chem B 4:2253-2263

18. Zhou Y, Kong Y, Kundu S, Cirillo JD, Liang H (2012) Antibacterial activities of gold and silver nanoparticles against Escherichia coli and bacillus Calmette-Guérin. J Nanobiotechnol 10(19):1-9

19. Li X, Robinson SM, Gupta A, Saha K, Jiang Z, Moyano DF, Sahar A, Riley MA, Rotello M (2014) Functional gold nanoparticles as potent antimicrobial agents against multi-drug-resistant bacteria. ACS Nano 8(10):10682-10686

20. Jain S, Hirst DG, O’Sullivan JM (2012) Gold nanoparticles as novel agents for cancer therapy. Br J Radiol 85(1010):101-113

21. Curry T, Kopelman R, Shilo M, Popovtzer R (2014) Multifunctional theranostic gold nanoparticles for targeted CT imaging and photothermal therapy. Contrast Media Mol Imaging 9(1):53-61

22. Basavegowda N, Kumar GD, Tyliszczak B, Wzorek Z, SobczakKupiec A (2015) One-step synthesis of highly-biocompatible spherical gold nanoparticles using Artocarpus heterophyllus Lam. (jackfruit) fruit extract and its effect on pathogens. Ann Agric Environ Med 22:84-89

23. He YQ, Liu SP, Kong L, Liu ZF (2005) A study on the sizes and concentrations of gold nanoparticles by spectra of absorption, resonance Rayleigh scattering and resonance non-linear scattering. Spectrochim Acta 61:2861-2872

24. Sobczak-Kupiec A, Malina D, Zimowska M, Wzorek Z (2011) Characterization of gold nanoparticle for various medical application. Dig J Nanomater Biostruct 6:803-808 